\title{
Curricular internationalization in higher education in Slovakia through the integration of content and language - experience with teaching in French
}

\section{[L'internationalisation curriculaire dans l'enseignement superieur en Slovaquie par l'integration du contenu et de la langue ; le cas des formations en français]}

\author{
Maria Rostekova - Maria Palova
}

DOI: 10.18355/XL.2020.13.01.16

\begin{abstract}
The goal of this paper is to determine how certain Slovak universities offering double degrees with French partners deal with the integration of content and language, and how they apply different educational approaches known by the acronym CLIL. As we had assumed, most of the teachers interviewed were not familiar with CLIL. They knew neither its theoretical foundations and principles nor its many possible implementations. Nevertheless, we have observed that many aspects of CLIL are used by pedagogues as part of their teaching. We have also examined the coherence between the approaches deployed by those teachers and the internationalization strategy recommended by the institutions where they teach.
\end{abstract}

Key words: CLIL, integration of content and language, curricular internationalization, higher education, double degree, questionnaire

\section{Résumé}

L'objectif de cet article est de déterminer comment certaines universités slovaques proposant des programmes doubles avec les partenaires français abordent l'intégration du contenu et de la langue et comment elles appliquent différentes approches éducatives, désignées par l'acronyme EMILE. Un questionnaire a été élaboré à cet objectif. Comme nous l'avions supposé, la plupart des enseignants interrogés n'étaient pas familiarisés avec cette approche. Ils ne connaissaient ni ses fondements théoriques et principes, ni ses différentes approches. Malgré cela, nous avons observé que de nombreux aspects d'EMILE sont pourtant mis à profit par les pédagogues dans le cadre de leur enseignement. Nous nous sommes aussi intéressées à la cohérence entre les approches déployées par ces enseignants et la stratégie d'internationalisation préconisée par les institutions qui les emploient.

Mots clés : EMILE, intégration du contenu et de la langue, internationalisation curriculaire, enseignement supérieur, formations doubles, questionnaire

\section{Introduction}

Le concept d'internationalisation a été, pendant longtemps, porteur de nombreuses significations, ce qui explique non seulement sa complexité et son développement constant, mais également ses différentes réalisations pratiques, rendues possibles par différents contextes culturels. Aujourd'hui, l'internationalisation est considérée comme l'un des outils les plus importants pour améliorer la qualité de l'enseignement supérieur, une attention accrue étant accordée à ce phénomène depuis la fin des années 1990 où l'internationalisation est devenue partie intégrante de l'évaluation nationale. Pendant cette période relativement courte, la diversification des politiques a été fondamentale et de nouvelles formes d'internationalisation de l'enseignement supérieur, notamment celles liées au contenu des programmes d'études, font l'objet d'un examen attentif. 
Dans de nombreux pays, y compris la Slovaquie, l'internationalisation a été largement influencée par le processus d'intégration européenne, les réformes apportées dans ce domaine étant directement liées à la mise en œuvre du processus de Bologne (Lips, 2016). L'enseignement supérieur européen radicalement transformé depuis son lancement et la création de programmes bilingues ou plurilingues qui favorisent l'enseignement de matières par l'intégration d'une langue étrangère, connu sous l'acronyme EMILE, en est un bon exemple. L'appropriation des langues étrangères est apparue comme un outil prioritaire accompagnant la transmission des savoirs d'un pays à l'autre. C'est dans ce contexte plurilingue et pluriculturel encourageant des échanges internationaux que la notion d'EMILE est apparue (Napoli, Sourisseau, 2013).

Ces transformations ont apporté, partout en Europe, de nouvelles perspectives de recherche sur l'enseignement et l'apprentissage par le biais d'une langue additionnelle, ce qui permet d'en étudier l'impact sur les programmes et formations universitaires existants, le développement professionnel des enseignants, sans oublier ce qui relève de l'assurance qualité. En Slovaquie, nous pouvons constater des changements curriculaires de même nature: les universités slovaques ont considérablement élargi leur offre par la création de programmes de licence et de master en langues étrangères, en particulier en anglais, qui est la lingua franca, la langue de la science et de la recherche et du marché mondialisé du travail. Cependant, l'internationalisation des programmes dans les universités slovaques se fait aussi en français. Il ne s'agit pas seulement des formations slovaques réalisées en langue française, mais aussi des programmes doubles, dispensés conjointement par les institutions partenaires dans les deux pays qui font objet de notre intérêt dans cet article.

Comptant parmi les premiers programmes doubles en Slovaquie, ces formations franco-slovaques sont proposées dans les domaines des sciences économiques, techniques et juridiques, mais aussi des relations internationales, des études européennes et de la traduction. Existant depuis une dizaine d'années, leur nombre et leur situation sont variables : certains projets ont été arrêtés alors que d'autres sont encore en cours de création (Rostekova, 2018). Le principal avantage de ces programmes est lié à l'intégration de tous les principes de base du processus de Bologne dans la pratique : plus grande mobilité, études structurées de façon similaire et processus de démarche qualité. Le fait que ces programmes soient réalisés via une autre langue étrangère que l'anglais contribue à atteindre l'un des objectifs de l'UE en soutenant le plurilinguisme.

L'objectif de cet article est de déterminer comment certaines universités slovaques proposant ces programmes abordent l'intégration du contenu et de la langue, c'est-àdire comment elles appliquent différentes approches éducatives, qui sont généralement désignées par l'acronyme «EMILE». Nous avons élaboré un questionnaire (avec des questions fermées et des questions ouvertes), afin de recueillir deux types de données : celles, objectives, sur les données quantifiables et celles, plus subjectives, liées aux prises de position et aux opinions. Nous supposons néanmoins que la majorité des enseignants interrogés n'est pas encore familiarisée avec ce qu'est EMILE, c'est-à-dire qu'elle ne connaît ni ses fondements théoriques et principes ni ses différentes approches. Malgré cela, nous estimons que de nombreux aspects de l'EMILE sont pourtant mis à profit par les pédagogues dans le cadre du processus d'enseignement, ces aspects faisant partie du cursus d'un programme d'études donné ou du syllabus d'une matière. Nous nous intéressons ici à la cohérence entre les approches déployées par certains pédagogues et la conception de l'internationalisation

XLinguae, Volume 13 Issue 1, January 2020, ISSN 1337-8384, eISSN 2453-711X 
en vigueur dans les institutions qui les emploient. Nous nous attendons néanmoins à ce que les réponses varient en fonction du degré d'internationalisation de chaque unité de travail et de la stratégie mise en place dans ce domaine.

\section{L'intégration du contenu et de la langue en théorie et pratique}

L'enseignement de matières par l'intégration d'une langue étrangère ou EMILE (en anglais Content and Language Integrated Learning, CLIL) s'inscrit dans l'un des courants éducatifs les plus importants de l'espace européen actuel. De par son caractère interdisciplinaire marqué, il permet de connecter l'enseignement des langues à celui d'une matière. L'objectif de ce type d'enseignement est une orientation équivalente du contenu et de la langue, ce qui le différencie d'un enseignement bilingue ou de l'enseignement de matières spécialisées en langue étrangère (souvent considéré à tort comme relevant de l'EMILE). Dans ces deux cas, l'attention se porte principalement sur un contenu enseigné uniquement en langue étrangère - ce qui implique des attentes certaines concernant le degré de fluidité dans l'emploi de la langue en question ${ }^{1}$. L'EMILE est aussi considéré relativement souvent en tant qu'enseignement d'une langue sur la base des thèmes d'une matière de spécialité autrement dit, sur la base de rapports entre matières où l'objectif principal n'est pas d'améliorer le niveau de langue, mais d'acquérir de nouvelles connaissances liées à une discipline non linguistique, présentée par le biais d'une langue étrangère.

D'un point de vue méthodologique, il existe, toutefois, une différente de taille entre, d'une part, les approches précédemment décrites et celles relevant de l'EMILE et, d'autre part, entre l'EMILE et l'enseignement des langues étrangères. Si ce dernier se consacre en grande partie à approfondir quatre compétences (compréhension et expression écrite et orale), l'enseignement d'une discipline non linguistique met en œuvre ces compétences afin d'obtenir de nouvelles informations et de vérifier leur bonne compréhension. C'est d'ailleurs cette compétence - la faculté d'agir en langue étrangère lors de diverses situations - qui peut être considérée comme le plus grand apport de l'EMILE, en particulier dans le cadre des futures professions des apprenants qui ne se contentent pas d'assimiler des connaissances linguistiques dont ils auront éventuellement besoin plus tard, mais disposent de savoir-faire pouvant être appliqués immédiatement.

Les origines de l'enseignement intégré d'un contenu et d'une langue datent approximativement de la seconde moitié du $20^{\mathrm{e}}$ siècle (Hanesova, 2015), mais le terme lui-même n'a été introduit que dans les années 1990 par les experts en linguistique appliquée et des spécialistes de l'éducation au sein du milieu éducatif européen qui ont largement contribué à l'épanouissement de cette approche (Coyle, 2008 ; Coyle et al., 2010 ; Perez-Canado, 2013). L'EMILE est devenu un réel domaine de recherche au début du nouveau millénaire, comme le prouvent les nombreux travaux au niveau mondial ou européen. Son utilisation est plus fréquente dans les établissements du primaire et du secondaire, ou le terme EMILE est lié à divers programmes éducatifs : enseignement bilingue, en immersion et plurilingue ou programmes renforcés au niveau linguistique (Hurajova, 2017). Cependant, il faut se rendre compte du fait que ces programmes peuvent ou non utiliser l'approche EMILE (Gabillon, 2019).

Ces derniers temps, l'EMILE est de plus en plus souvent utilisé dans l'enseignement tertiaire, où derrière cette appellation peuvent se cacher des pratiques pédagogiques

\footnotetext{
${ }^{1}$ L'objectif principal de ce type d'enseignement n'est pas d'améliorer le niveau de langue, mais surtout d'acquérir de nouvelles connaissances liées à une discipline non linguistique, présentée par le biais d'une langue étrangère.
} 
très différentes. Pour le seul espace anglophone, on trouve une multiplicité de dénominations : Content-Based Instruction (CBI), Content-Based Learning (CBL), Language for Specific Purposes (ESP) (avec toutes ses dérivations), Cognitive Academic Language Learning Activities (CALLA), Task-Based Learning (TBL), English-medium Instruction (EMI), Teaching Content through a Foreign Language (TCFL), English-medium Masters (EMM), International Master's Programmes (IMP) etc. Ces approches peuvent être regroupées car elles partagent certaines hypothèses, à savoir : l'enseignement / l'apprentissage d'une langue étrangère est une pratique éducative ; le contenu est indissociable de l'expression linguistique ; il est nécessaire de coordonner l'apprentissage de la langue et de la matière enseignée ; la langue est le principal moyen d'instruction et d'apprentissage; le contenu de la matière contextualise l'apprentissage des langues (Gonzalez Ardeo, 2013).

À la différence d'autres pays européens, les recherches relatives à l'EMILE en sont encore à leurs balbutiements en Slovaquie (Hurajova, 2017). L'attention des spécialistes slovaques se porte en particulier sur l'enseignement secondaire notamment les écoles bilingues - ainsi que sur les établissements "classiques" mettant en œuvre les principes de l'EMILE. En revanche, tout reste à faire au niveau de l'enseignement tertiaire ${ }^{2}$. Parmi les auteurs qui se sont penchés sur la problématique, citons Maria Badinska (2011), ainsi que le duo Jana Luprichova et Ludmila Hurajova (2017) qui, dans le cadre d'un projet institutionnel réalisé à la Faculté des Sciences des matériaux et technologies de l'Université technique slovaque ont analysé l'attitude des enseignants lors de l'enseignement de cours théoriques en anglais parallèlement à leur préparation linguistique ${ }^{3}$.

\section{Quelle approche de l'EMILE dans l'enseignement supérieur ?}

Au niveau universitaire partout dans le monde, nous avons pu observer un certain virage institutionnel effectué dans le domaine des langues sur objectifs spécifiques (LOS) pour aboutir à diverses formes d'EMILE où l'offre de cours est adaptée de sorte que celle-ci corresponde mieux aux attentes des apprenants vis-à-vis d'une discipline donnée. Forts de ces observations, les spécialistes d'EMILE sont prêts à collaborer avec les enseignants chargés de créer les contenus pédagogiques afin d'améliorer la compétence des diplômés en matière d'enseignement en langue étrangère (Arno-Macia, Mancho-Bares, 2015). Ces pratiques ont rompu avec la traditionnelle et arbitraire division curriculaire par matières tout en favorisant une intégration curriculaire plus pertinente des domaines linguistiques et non linguistiques en lien avec les objectifs, les processus d'apprentissages et les procédures d'évaluation.

C'est ainsi l'intégration curriculaire qui se révèle primordiale en matière d'EMILE, car elle requiert l'acquisition simultanée de contenus de nature linguistique et non

\footnotetext{
${ }^{2}$ Plusieurs auteurs slovaques, en revanche, se recentrent sur la langue sur objectif spécifique (LOS) et parmi eux, nous voulons citer les suivants : M. Badinska (2013), M. Bencekova (2019), R. Gura (2005), V. Kresakova - J. Pecnikova, (2019), J. Kubekova (2017), E. Molnárová (2016), M. Polcicova (2018), Zelenkova Spisiakova (2009), etc.

${ }^{3}$ Le projet prévoit la création de cellules EMILE pluridisciplinaires dans le cadre duquel le personnel enseignant devrait être formé sur les plans linguistique et didactique afin de lancer ses cours en anglais, étape par étape. Des enseignants de l'ESP devraient être membres de ce groupe de cellules EMILE afin d'aider d'autres enseignants à préparer leurs cours en anglais.
}

XLinguae, Volume 13 Issue 1, January 2020, ISSN 1337-8384, eISSN 2453-711X 
linguistique (Martin de Lama, 2015). Cependant, elle présuppose des finalités différentes pour les contenus pédagogiques et les contenus langagiers, sachant qu'une langue est perçue très diversement selon les personnes et le contexte : si la langue représente un outil d'interaction et de communication stratégique pour chacun de ses utilisateurs, elle constitue en revanche pour les enseignants et apprenants une matière (ou un contenu) destinée à être enseignée et étudiée ; pour les linguistes, il s'agira d'une discipline et d'un objet de recherche et pour les professionnels du milieu académique, la langue constituera un outil et un intermédiaire visant à construire des connaissances tout en permettant un échange de savoirs.

Il est important que les spécialistes de chaque discipline et les experts en langue définissent de la même façon ce que des termes comme "langue » et "acquis 》 doivent signifier dans un contexte d'EMILE. L'apprentissage d'une langue par EMILE doit donc être appréhendé de sorte que la fonction donnée aux acquis d'apprentissage de la langue soit en adéquation avec le Cadre Européen Commun de Référence pour les Langues (Martin de Lama, 2015). Ce mécanisme d'intégration de l'apprentissage d'une langue étrangère aux contenus curriculaires non linguistiques implique une mosaïque complexe de langues et des techniques et mécanismes de raisonnement qui permettront d'augmenter la motivation des apprenants et de favoriser l'amélioration simultanée de la maîtrise de la langue cible, des compétences cognitives et de la compréhension des contenus.

L'émergence de l'EMILE dans l'environnement de l'enseignement supérieur fait, toutefois, remonter un grand nombre de questions liées à la manière dont cette pratique pourrait être appliquée graduellement à des contextes d'apprentissage médiés par la langue - en particulier dans l'organisation et les procédures aux différents niveaux pédagogiques, vis-à-vis notamment des programmes d'études lors de remaniements méthodologiques et linguistiques destinés à satisfaire aux besoins des apprenants (Martin de Lama, 2015). Pour ces raisons, l'EMILE doit être considéré comme une continuité de différentes approches pédagogiques ayant pour but de faciliter l'apprentissage.

De nombreuses initiatives ont déjà été lancées par des institutions supérieures partout dans le monde afin de renforcer l'apprentissage des langues, même si les résultats attendus ne restaient exprimés qu'implicitement dans les approches. En fonction du degré d'intégration curriculaire et du type de focalisation (sur la langue ou sur la matière étudiée), mais aussi du nombre d'heures d'instruction réalisée par immersion, ces initiatives supposent un large éventail de contextes éducatifs dans un continuum allant d'une approche « douce » ou « appuyée » en passant par un EMILE modulable (Martin de Lama, 2015).

La meilleure analyse de cette oscillation, pour l'objectif de la présente contribution, est celle fournie par A. Greere et A. Rasanen (2008) qui proposent une classification permettant de distinguer des palettes de cours allant d'une absence totale d'intégration de la langue et du contenu à des situations de collaboration idéale entre spécialistes de langues et leurs collègues de disciplines non linguistiques. Leurs approches pourraient être décomposées de la façon suivante :

1. NON-EMILE signifie qu'il n'y a pas réellement d'intérêt pour l'apprentissage de la langue et la collaboration (pédagogique) y est inexistante. L'exposition des apprenants à la langue reste secondaire, non systématique et limitée $(<25 \%)$.

2. L'approche dite PRÉ-EMILE (langue sur objectif spécifique) peut être appréhendée comme un enseignement de langue basé sur une discipline : des spécialistes de langue y fournissent des enseignements calibrés afin de 
faciliter l'apprentissage de la discipline, mais il n'y a pas de collaboration (systématique) avec les spécialistes du domaine étudié. L'enseignant de langue choisit lui-même son matériel.

3. Dans l'EMILE partiel basé sur la langue, l'enseignement linguistique préalable ainsi que l'étude du discours et des pratiques académiques sont primordiaux afin de favoriser l'apprentissage des contenus d'un cours/programme par les étudiants. La collaboration est possible entre enseignants et les acquis d'apprentissage de la langue sont définis par les besoins des contenus enseignés.

4. Dans l'EMILE partiel basé sur le contenu, les cours/programmes sont systématiquement dispensés par des spécialistes du domaine à des groupes mixtes, multiculturels et multilingues ( $>25 \%$ d'exposition). L'apprentissage de la langue est effectué en raison de l'exposition au langage, mais les résultats attendus ne sont pas spécifiés, les objectifs et les critères étant seulement implicites. Si la collaboration est possible, elle reste, toutefois, rare.

5. L'EMILE adjoint suppose un soutien linguistique coordonné et intégré aux matières étudiées. L'ensemble des cours a lieu au même endroit et le planning est réalisé en commun par les enseignants. Les critères et les résultats attendus sont spécifiés pour la langue et la spécialité.

6. Pour conclure, l'EMILE constitue une approche totalement duelle avec intégration complète de la langue au domaine étudié par les enseignants de la spécialité ou l'ensemble de l'équipe enseignante. Les critères et les résultats attendus sont spécifiés pour la langue et la spécialité (Greere, Rasanen, 2008).

Il convient encore de souligner les raisons pour lesquelles les approches EMILE sont appliquées dans l'enseignement supérieur. Si la principale motivation menant à l'utilisation de l'EMILE est le désir d'améliorer des compétences en langue en élargissant le champ traditionnel de l'enseignement des langues étrangères tout en atteignant parallèlement le même degré de spécialisation que si le cours avait été dispensé dans la langue maternelle de l'étudiant, il y a toutefois d'autres attentes qui sont associées à l'EMILE, à savoir : améliorer la compréhension interculturelle et la sensibilisation aux langues ; approfondir le degré d'apprentissage de la matière grâce à une stimulation cognitive ; offrir l'accès à des référentiels de connaissance disponibles en d'autres langues ; proposer une approche didactique innovante plus centrée sur l'apprenant et dépasser les traditionnelles frontières entre matières, pour n'en nommer que certaines, ou encore mieux préparer les étudiants à leur carrière professionnelle à l'heure de la mondialisation (Dalton-Puffer, 2019). L'une des principales tâches des recherches dédiées à l'EMILE consiste, donc, à déterminer dans quelle mesure ces hypothèses positives peuvent être étayées par des observations concrètes liées à une pratique réelle de l'EMILE.

Il est également important de réaliser que la langue dans laquelle l'EMILE est appliqué bénéficiera d'un impact déterminant en matière de connaissance préalable, de motivation et de point de vue des personnes concernées ainsi que sur le résultat. Si en théorie l'EMILE peut se faire dans une variété de langues, en pratique la langue qui fait l'objet de l'intégration au contenu est le plus souvent l'anglais. Néanmoins, le statut prédominant de lingua franca mondiale signifie que toutes les affirmations concernant l'anglais ne peuvent être appliquées automatiquement et sans questionnement préalable à toute autre langue d'EMILE. On ne peut par exemple exclure que certains «succès» d'EMILE soient liés aux bénéfices réels ou 
imaginaires d'une bonne maîtrise de l'anglais plutôt qu'à l'approche EMILE à proprement parler.

En bref, la réalité de l'EMILE peut prendre différentes formes, allant de brèves phases de projet à l'enseignement de presque toutes les matières dans la langue d'EMILE. Pour cette raison, les chercheurs doivent, selon C. Dalton-Puffer (2019), non seulement réfléchir à ce que toutes les formes d'EMILE ont en commun (une double attention portée sur la langue et objectifs en matière de contenus d'apprentissage dans la salle de classe), mais aussi tenir compte des différents cadres institutionnels dans lesquels sont réalisés les programmes EMILE spécifiques.

\section{Synthèse des résultats du questionnaire complété par les enseignants}

Notre étude a pour objet de délimiter les « formes » communément regroupées sous le terme «EMILE» dans le cadre des programmes d'études conjoints (que la terminologie française qualifie de "programmes doubles ») mis en place par les universités slovaques en collaboration avec des partenaires français. Cinq établissements slovaques - tous membres de 1'Institut universitaire franco-slovaque $(\mathrm{IUFS})^{4}$ - réalisent actuellement ce type de coopération internationale. Ce soutien permet notamment à 12 programmes d'études franco-slovaques (principalement de deuxième cycle) d'exister tout en bénéficiant de l'accréditation des ministères de l'Éducation français et slovaque.

Les programmes d'études conjoints représentent le plus haut niveau de coopération internationale et leur apport s'avère important pour l'internationalisation de l'enseignement supérieur et le développement de l'enseignement tertiaire en Slovaquie : outre une augmentation de la mobilité des étudiants et enseignants, les programmes mentionnés permettent de consolider la coopération (pédagogique et scientifique) internationale des institutions participantes et d'interconnecter les contenus des différents programmes d'études tout en accroissant les possibilités de reconnaissance mutuelle des diplômes (Rostekova, 2018).

L'offre de programmes conjoints vient également renforcer la réputation d'une université à l'international, permet de s'orienter vers certains domaines difficiles d'accès, soutient la coopération internationale au niveau de l'enseignement et augmente la capacité d'une institution à rapidement réagir à de nouveaux besoins (Soltes, Neupauerova, 2008). Ces programmes aboutissent également à une plus grande transparence des systèmes d'enseignement et amènent à modifier certaines normes juridiques au niveau national. Dans un contexte de crise démographique et d'exode massif des jeunes Slovaques vers des universités étrangères (principalement en République tchèque), ces programmes viennent renforcer l'attractivité des universités slovaques, devenant ainsi un outil important en vue de retenir les étudiants dans nos établissements (Mortreuil, 2017).

Notre enquête a été réalisée par questionnaire auprès de trois universités membres de l'IUFS, à savoir l'Université Matej Bel de Banska Bystrica (UMB), l'Université Pavol Jozef Safarik de Kosice (UPJS) et l'Université technique de Kosice (TUKE), toutes

\footnotetext{
${ }^{4}$ Les deux auteures du présent article sont membres actives de l'IUFS : Maria Palova préside le comité du directeur de l'Institut tandis que Maria Rostekova est membre-fondatrice. Les deux enseignantes coordonnent ces programmes dans leur université d'origine et participent à leur promotion au niveau national ainsi qu'à la mise en place de nouvelles formes de coopération à l'échelle nationale. Cf : https://www.iufs.sk/sk/univerzita/studijne-programy
} 
les trois s'efforçant de créer un environnement favorable et une expérience éducative qui préparent les étudiants à devenir des citoyens d'une société multiculturelle à la fois dans leur pays, mais également à l'échelle européenne et mondiale.

Nous avons recueilli les réponses de 57 enseignants aux profils et expériences pédagogiques des plus variés. Si nous avons d'abord privilégié des personnes enseignant la langue française ou en français, nous avons ensuite élargi notre groupecible en nous adressant à d'autres pédagogues d'une section (département) donnée, car ceux-ci participent également à la conception d'un programme d'études commun aux côtés de partenaires français - ou bien enseignent au sein d'un autre programme conjoint - car leur expérience peut avoir une incidence, directe ou indirecte, sur la conception des programmes d'études communs en langue française. En outre, de nombreux programmes francophones sont réciproques, ce qui signifie que les étudiants inscrits effectuent une partie de leur cursus dans une université partenaire. Une partie des enseignements suivis par les étudiants francophones en milieu universitaire slovaque est dispensée en anglais - cela concerne notamment les matières du «tronc commun » proposées par des universitaires non francophones.

Actuellement, deux composantes de l'UMB proposent des programmes d'études conjoints en collaboration avec des partenaires français : la Faculté d'Économie et la Faculté des sciences politiques et des relations internationales. Les programmes d'études francophones possèdent une longue tradition au sein des deux facultés puisqu'ils ont été mis en place au début des années 1990, d'abord sous la forme de cours de français sur objectifs spécifiques, puis de cours de spécialité dispensés en langue française, voire de modules complets proposés dans le cadre de certains programmes d'études (Horehajova, Marasova, 2017).

L'acquisition d'expériences et le développement d'un réseau de partenaires étrangers par le biais de cette initiative ont progressivement débouché sur les premiers programmes d'études conjoints en langue française. Le programme de licence «Gestion et Communication» a été ouvert en 2007 à la Faculté d'Économie en partenariat avec l'Université de Poitiers et a été suivi en 2010 par le programme de master «Gestion financière et espace européen » réalisé avec l'Université de Lorraine Metz-Nancy. Le dernier-né date de 2012 : il s'agit du master « Droit des collectivités locales et des entreprises culturelles » mis en place avec l'Université de Reims Champagne-Ardenne.

La Faculté des sciences politiques et des relations internationales a proposé son premier programme d'études conjoint, le master «Spécialistes de l'intégration communautaire et de la politique européenne de voisinage » en 2008, en collaboration avec l'Université de Reims Champagne-Ardenne. Son offre a été renforcée quelques années plus tard par les programmes de master "Analyse des conflits et de la violence » mis en place avec l'Université de Versailles-Saint-Quentin-en-Yvelines et «Géopolitique» avec l'Université de Reims Champagne-Ardenne. La Faculté ne propose actuellement que le premier des programmes mentionnés sous l'intitulé « Intégration européenne et gouvernance globale ».

Nous avons également étendu notre enquête à la Faculté des Lettres de l'UMB, concrètement au Département d'Études culturelles européennes, et ce même s'il ne propose pas à proprement parler de programme d'études conjoint. La licence et le master du programme «Études culturelles européennes » représentent néanmoins la plus haute forme d'internationalisation curriculaire, désignée par le terme « internationalisation à domicile »: grâce aux langues étrangères, les étudiants se

XLinguae, Volume 13 Issue 1, January 2020, ISSN 1337-8384, eISSN 2453-711X 
familiarisent durant leurs études avec quatre cultures dominantes de l'Europe contemporaine. Les apprenants choisissent habituellement deux cultures « majeures » à étudier en langue étrangère, les deux autres étant étudiées en slovaque lors du cycle de licence. Le master se poursuit ensuite avec l'étude des deux «majeures » en langues étrangères.

Le développement des programmes d'études conjoints du cycle d'études de master et d'études doctorales compte parmi les axes prioritaires de la coopération internationale de la Faculté des Lettres de l'UPJS de Kosice. Au total, douze programmes d'études conjoints sont mis en œuvre dont neuf sous la gestion du Département d'études britanniques et américaines. Deux universités françaises sont concernées : l'Université de Strasbourg pour le programme d'études de master en traduction professionnelle (depuis 2016) et l'Université Paris Diderot pour le programme d'études doctorales en linguistique de corpus (depuis 2013). Même si la Faculté des Lettres n'a pas de Département de français, dans les deux programmes visés le français et l'anglais sont présents en proportions équilibrées. De plus, en traduction professionnelle, durant deux semestres d'études obligatoires à Strasbourg, le français devient langue A, c.-àd. "langue maternelle » des étudiants slovaques. Dans les deux langues sont enseignées les connaissances thématiques (droit, économie, institutions européennes, théories linguistiques ou traductologiques); et certaines compétences, telles que les outils informatiques, la création et exploitation de corpus multilingues, les outils de la traduction, la traduction automatique ou la post-édition sont acquises en fonction de la langue disponible de l'un ou de l'autre établissement partenaire.

La Faculté d'Économie de la TUKE propose un programme d'études conjoint en collaboration avec son partenaire français - l'Université de Côte d'Azur. Le programme d'études francophones possède une longue tradition, il a commencé d'abord sous forme de cours de français sur objectifs spécifiques (FOS) à partir de 2003 puis, à partir de 2004, de cours de spécialité en langue française. Le programme d'études conjoint en « Économie et Gestion » est mis en place au niveau de la licence.

Le questionnaire distribué se composait de trois parties. La première nous a permis de recueillir certaines données personnelles relatives aux répondants, en particulier la durée de leur expérience ainsi que quelques précisions quant à leur profil pédagogique. Nous avons considéré qu'il était nécessaire de distinguer les professeurs de langues étrangères de ceux dispensant des cours de spécialité en langue étrangère, cette question étant principalement liée à la spécialisation du département donné. Ces deux critères nous ont permis de délimiter les cinq catégories suivantes : a) enseignant de langue étrangère au sein d'une section d'orientation philologique, b) enseignant de langue étrangère dans une section d'orientation non philologique, c) enseignant de spécialité en langue étrangère d'une section d'orientation philologique, d) enseignant de spécialité en langue étrangère d'une section d'orientation non philologique, e) enseignant de langue étrangère dispensant également des cours de spécialité en langue étrangère. Nous nous sommes également intéressées au degré de participation des répondants aux programmes d'études conjoints - non seulement à ceux réalisés avec des partenaires français, en langue française, mais aussi à des cursus proposés via d'autres langues. Nous avons supposé que les réponses d'enseignants rompus à diverses formes de collaboration internationale correspondraient plus précisément au principe de l'approche EMILE.

La seconde partie du questionnaire nous a permis d'observer comment se réalise l'intégration de la langue et du contenu lors de la pratique pédagogique des répondants. L'objectif était d'évaluer l'importance accordée à une acquisition 
pondérée et simultanée par les étudiants d'un contenu de spécialité et d'une langue étrangère - ce qui correspond aux principaux traits de l'approche EMILE - ou de constater, au contraire, une primauté de la langue ou du contenu de spécialité. En d'autres termes, nous avons cherché à savoir si les enseignants privilégient une forme «douce» ou «dure » de l'approche. Nous nous sommes également intéressées au degré de collaboration entre les enseignants de langue et ceux de spécialités dispensées en langue étrangère, aussi bien lors de la conception et la réalisation d'une matière donnée que pendant l'évaluation des connaissances et du savoir-faire des étudiants. Une autre question d'importance est celle de l'utilisation de niveaux de références en langue et de descripteurs visant à définir les objectifs et modes d'évaluation liés aux standards de compétence et de contenu définis par le Cadre européen commun de référence pour les langues (CECRL). Pour conclure, nous avons analysé la manière dont les enseignants appréhendent leur propre processus d'enseignement dans un contexte EMILE. Les cinq possibilités de réponse proposées reprenaient différentes approches décrites par A. Greere et A. Räsänen dans leur classification (Non-EMILE, Pré-EMILE, EMILE partiel basé sur la langue, EMILE partiel basé sur le contenu, EMILE adjoint et EMILE total et complet) que nous avons considérée comme étant la plus adaptée à la situation actuelle de l'enseignement supérieur slovaque. Cf. Tableau $n^{\circ} 1$. Outre les possibilités de réponses proposées dans cette section du questionnaire, il nous a semblé indispensable d'utiliser des questions ouvertes afin de mieux cerner la réalité de la pratique pédagogique de chaque enseignant interrogé.

La dernière partie de notre enquête était consacrée à l'internationalisation des institutions employant les répondants. Nous avons cherché à savoir si les pédagogues étaient au fait de la stratégie d'internationalisation mise en place par leur employeur tout en leur demandant s'ils estiment que la matière qu'ils enseignent (langue étrangère ou spécialité en langue étrangère) correspond aux objectifs d'internationalisation de leur département et si cette stratégie influence l'amélioration de la qualité du cursus du domaine et le syllabus de leur matière (introduction d'une nouvelle méthodologie, définition de nouveaux objectifs pour la matière, modifications du contenu enseigné, etc.). Nous avons également alterné questions fermées et ouvertes dans cette partie.

Nous avons distribué un total de 80 questionnaires pour les besoins de notre enquête. 57 nous ont été retournés complétés, 53 étant considérés comme exploitables. Les réponses liées à la durée de l'expérience pédagogique (une seule réponse possible) et au profil (deux réponses maximum) nous ont fourni un large éventail d'informations. 41 pédagogues ont mentionné une longue carrière dans l'enseignement -22 indiquant exercer leur activité depuis 11 à 20 ans et 19 depuis plus de 20 ans. Trois autres répondants enseignent depuis 2 à 5 ans et un depuis 6 à 10 ans. Une seule personne a mentionné une expérience inférieure à 2 ans.

La majorité des répondants a fait état d'une spécialisation liée à un seul domaine. Il s'agit concrètement d'enseignants de matière de spécialité en langue étrangère issus de sections d'orientation non philologique, au nombre de 20. Nous pouvons ajouter à ce groupe trois personnes qui enseignent également une matière de spécialité d'orientation philologique et deux autres qui dispensent aussi des cours de langue étrangère au sein d'un département d'orientation non philologique. La seconde catégorie la plus nombreuse est celle des enseignants de langue étrangère employés par des départements d'orientation non philologique qui représente un total de 14 personnes, dont 6 qui enseignent également une matière de spécialité en langue étrangère. Nous n'avons dénombré que 8 enseignants de langue étrangère issus de

XLinguae, Volume 13 Issue 1, January 2020, ISSN 1337-8384, eISSN 2453-711X 
départements d'orientation philologique, dont une personne dispensant parallèlement des cours de spécialité en langue étrangère dans un département d'orientation non philologique. 8 répondants ont indiqué être à la fois enseignants de langue étrangère et de matières de spécialité en langue étrangère.

Si l'on s'intéresse au profil linguistique, le français a été mentionné par 11 personnes enseignant cette langue ou en utilisant cette langue, ce qui représente $19 \%$ du total des répondants. Parmi eux, 6 ont indiqué une seconde langue (l'anglais étant majoritaire). $41 \%$ des enseignants interrogés ont pour leur part répondu en mentionnant la langue anglaise, dont 7 personnes utilisant celle-ci en combinaison avec une autre. La part des autres langues est restée relativement faible.

À la question relative au degré de connaissance de l'enseignement de matières par l'intégration d'une langue étrangère (EMILE), seules 33 personnes ( $40 \%$ du total) ont répondu par l'affirmative, 13 d'entre elles (24\%) indiquant utiliser l'approche dans le cadre de leur pratique pédagogique. 8 autres enseignants ont indiqué connaître l'EMILE, mais uniquement d'un point de vue théorique. 18 répondants ont indiqué n'avoir jamais entendu parler de la méthode, ce qui représente $33 \%$ de l'ensemble des personnes interrogées (il s'agit majoritairement d'enseignants de matières de spécialité en langue étrangère). 12 personnes ont, quant à elles, indiqué avoir entendu parler de l'approche, mais sans expérience pratique ou théorique. Parmi les enseignants ayant affirmé connaître l'EMILE, 5 ont indiqué avoir rencontré l'approche au cours de leurs études universitaires et 6 dans le cadre de leur formation continue, 17 ont indiqué s'être familiarisé de façon individuelle, dans le cadre de travaux scientifiques ou d'activités liées à un projet.

Toujours en lien avec cette question, seules trois personnes enseignant en français ont affirmé utiliser l'approche dans la pratique - ces trois enseignants indiquent également enseigner par le biais d'une autre langue. Quatre répondants enseignant en français connaissent la méthode, mais ne possèdent aucune expérience y liée, un seul affirmant être familier des fondements théoriques de l'approche. Le plus grand écart a été constaté en lien avec cette première question (personnes n'ayant pas encore entendu parler de l'EMILE) et concerne le pourcentage distinguant les enseignants utilisant l'anglais de ceux utilisant le français. Il convient néanmoins de constater qu'il s'agit principalement d'enseignants de matières de spécialité.

Concernant l'analyse du degré d'intégration de l'enseignement de langue et de spécialité, 26 répondants ont affirmé veiller autant à l'acquisition du contenu de spécialité qu'au développement des compétences en langue étrangère. On trouve parmi eux 4 enseignants qui ne mentionnent pas de langue étrangère dans leur domaine de spécialisation. 22 répondants affirment se concentrer principalement sur l'acquisition des savoirs en langue étrangère liés à leur spécialité, 18 d'entre eux enseignant des matières de spécialité sans formation pour l'enseignement en langue étrangère. Seuls cinq enseignants indiquent accorder un poids plus important au développement des compétences communicationnelles, toutes ces personnes étant des enseignants de langue étrangère. Une importance égale accordée au contenu et à la langue est mentionnée par 9 répondants enseignant en français, trois d'entre eux indiquant posséder une seconde langue d'enseignement. Ce pourcentage est plus élevé que chez les répondants enseignant en anglais qui insistent principalement sur l'acquisition de nouvelles connaissances liées à la spécialité.

Les questions s'intéressant à la collaboration entre enseignants de langue étrangère et de spécialité permettent d'apprendre qu'environ $50 \%$ de tous les répondants estiment collaborer lors de la conception et de la réalisation d'une matière. Dans le cas des 
enseignants de langue étrangère, $41 \%$ ont affirmé être plutôt d'accord, $27 \%$ être totalement d'accord, $14 \%$ être sans avis tranché et $18 \%$ ont estimé être plutôt en désaccord sur cette question. La situation des enseignants de matières de spécialité en langue étrangère s'avère assez différente puisque près de $42 \%$ des répondants ont indiqué être en désaccord, $21 \%$ plutôt en désaccord et $8 \%$ sans opinion. Seuls $31 \%$ ont émis un avis positif. De leur côté, 6 enseignants de matières de spécialité en français se sont estimés satisfaits de leur collaboration, ce degré étant proportionnellement bien plus important que celui de leurs collègues enseignant en anglais qui ont majoritairement exprimé leur désaccord (15 répondants). La situation des enseignants de français langue étrangère (d'orientation philologique ou non) est assez similaire, le degré de satisfaction étant là aussi plus important que celui exprimé par leurs collègues enseignant l'anglais comme langue étrangère.

Pour ce qui est de l'évaluation des étudiants en cours de semestre et à la fin de celuici, la question du désaccord relatif à la collaboration a donné les résultats suivants : $80 \%$ des enseignants estiment ne pas collaborer dans ce domaine, $60 \%$ indiquant ne pas le faire du tout. Dans le cas des enseignants de langue étrangère, le degré d'insatisfaction est comparable puisque $43 \%$ ont indiqué ne pas collaborer du tout, $35 \%$ ont indiqué ne plutôt pas collaborer et $9 \%$ se sont déclarés satisfaits. Du côté des enseignants de spécialité en langue étrangère, $70 \%$ ont fait état d'une collaboration inexistante, $11 \%$ ont estimé ne pas vraiment collaborer et seuls $13,5 \%$ se sont déclarés satisfaits. La situation des enseignants utilisant le français est comparable à celle du cas précédent.

Concernant l'évaluation des aptitudes linguistiques des étudiants par le biais de niveaux de référence et de descripteurs émanant du CECR, 24 répondants ont affirmé utiliser ces outils tandis que 21 ne les utilisent pas - 14 d'entre eux étant des enseignants de matières de spécialité en langue étrangère. Trois personnes n'ont pas exprimé leur point de vue. Trois enseignants utilisant le français affirment avoir recours à des niveaux et descripteurs, l'un d'entre eux enseignant également en anglais. Les données relatives à cette question sont donc une nouvelle fois plutôt favorable aux enseignants utilisant le français.

Tous les pédagogues interrogés ont dû ensuite mentionner un maximum de deux traits caractéristiques correspondant à leur pratique d'enseignement parmi cinq descriptions proposées - sans pour autant savoir à quelle approche correspondait les réponses possibles. Seuls trois enseignants ont mentionné l'approche définissant un EMILE total et complet, deux d'entre eux sont des enseignants de langue étrangère sans orientation philologique et le troisième enseigne à la fois une langue étrangère sans orientation philologique et une matière de spécialité. Huit autres enseignants ont pointé conjointement la variante correspondant à un EMILE total et complet et celle de l'EMILE adjoint (il s'agit principalement d'enseignants de langue étrangère sans orientation philologique, trois d'entre eux dispensant également des cours de spécialité). Quatre autres personnes ont indiqué une combinaison d'un EMILE total et complet et d'un EMILE partiel basé sur le contenu (trois d'entre elles enseignant une matière de spécialité). La majorité des répondants (18 personnes) a choisi une seule caractéristique, celle de l'EMILE basé sur le contenu, parmi eux, 15 enseignent une matière de spécialité sans orientation philologique et 2 une matière d'orientation philologique. Les enseignants utilisant le français ont, pour leur part, indiqué les caractéristiques suivantes : 7 personnes ont mentionné l'EMILE basé sur le contenu (trois d'entre elles affirmant également utiliser l'EMILE total et complet). Deux répondants utilisant la même langue ont quant à eux cité l'EMILE partiel basé sur la langue.

XLinguae, Volume 13 Issue 1, January 2020, ISSN 1337-8384, eISSN 2453-711X 
La partie du questionnaire consacrée à l'internationalisation des départements a donné les résultats suivants : à la question interrogeant les enseignants sur leur connaissance d'une stratégie d'internationalisation, 2 répondants ont estimé ne pas être informés du tout et 8 ne pas être suffisamment informés tandis que 21 se sont estimés plutôt informés et 6 ont exprimé leur entière satisfaction quant au degré d'information. Les autres répondants n'ont pas exprimé d'opinion sur ce sujet. Interrogés ensuite sur la correspondance entre leur matière et les objectifs d'internationalisation de leur département, 34 enseignants ont exprimé leur satisfaction (23 plutôt satisfaits et 11 très satisfaits). Seuls trois répondants ont exprimé une opinion négative. À la dernière question qui cherchait à sonder si cette stratégie d'internationalisation avait un quelconque effet sur l'amélioration de la qualité curriculaire du domaine et le syllabus des matières, 29 personnes ont répondu par l'affirmative et 6 par la négative, 15 personnes ne s'exprimant pas. La matière enseignée et la langue employée n'ont néanmoins joué aucun rôle particulier lors des réponses à ces trois dernières questions.

\section{Conclusion}

Dans le contexte actuel d'internationalisation curriculaire du milieu universitaire, de plus en plus de formations de différentes disciplines sont dispensées en langue étrangère un peu partout dans le monde. L'anglais est majoritairement utilisé, mais d'autres langues peuvent également trouver leur place. Si l'enseignement de matières par intégration d'une langue étrangère, connu sous l'acronyme EMILE, ne représente qu'une des nombreuses formes d'internationalisation des formations, ce sujet donne lieu à des débats pédagogiques, scientifiques et politiques : la mise en place de cours en langues étrangères implique un véritable enjeu stratégique pouvant occasionner de nombreux avantages, notamment concernant l'attractivité des formations, l'intensification de la mobilité et l'amélioration de l'employabilité des étudiants, ce qui a pour effet d'accentuer les efforts institutionnels. Cette volonté doit néanmoins tenir compte du principal objectif de ces approches - l'amélioration substantielle de l'apprentissage des étudiants - les résultats de recherche effectués dans ce domaine devraient donc être pris en compte.

Cependant, à la différence d'autres pays, les recherches relatives à l'EMILE n'en sont qu'à leurs débuts en Slovaquie, l'attention des spécialistes slovaques se portant uniquement sur l'enseignement secondaire. Tout reste en revanche à faire concernant l'enseignement tertiaire. Cette raison nous a incitées à étudier l'intégration du contenu et de la langue par les enseignants des universités slovaques afin de savoir de quelle façon les approches pédagogiques de type EMILE étaient mises en place. Nous nous sommes focalisées sur les institutions proposant des programmes d'études doubles (conjoints) avec des partenaires français. Coordonnés par l'Institut universitaire franco-slovaque (IUFS), dont nous sommes membres, ces formations permettent un renforcement considérable de la réputation de l'enseignement supérieur slovaque à l'international.

Nous nous attendions à ce que l'absence de recherche dans ce domaine occasionne un manque de coordination au niveau pédagogique, ce qui se reflète dans le large éventail de formes d'EMILE. Si nous supposions que la majorité des enseignants interrogés n'était pas encore familiarisée avec cette méthode (c'est-à-dire qu'elle ne connaissait ni ses fondements théoriques et principes ni ses différentes approches), nous étions néanmoins persuadées que de nombreux aspects de l'EMILE étaient déjà mis à profit par ces pédagogues.

Nous avons effectué une enquête par questionnaire auprès de pédagogues enseignant le français ou en français (19\% des répondants), mais également d'autres personnes 
enseignant principalement en anglais, provenant de trois universités membres de l'IUFS, notamment de l'Université Matej Bel de Banska Bystrica (UMB), de l'Université Pavol Jozef Safarik de Kosice (UPJS) et de l'Université technique de Kosice (TUKE). Les résultats de l'enquête nous ont permis d'apprendre que le profil pédagogique de chaque enseignant jouait un rôle-clé vis-à-vis de sa connaissance des approches de l'EMILE et de l'utilisation pratique de celles-ci. La majorité des enseignants de matières de spécialité en langue étrangère (répondants spécialisés dans un seul domaine) ne connaît pas du tout la méthode et n'en a même jamais entendu parler. Ceux qui enseignent une langue étrangère parallèlement à une matière de spécialité constituent une exception quelle que soit l'orientation (philologique ou non) de leur département. Dans le cas des professeurs de matières de spécialité n'ayant pas bénéficié d'une formation d'enseignant en langue étrangère, la langue employée constitue un facteur-clé - les enseignants recourant au français apparaissent comme plus au fait des principes de l'EMILE que leurs collègues enseignant en anglais. Nous estimons que cette différence est notamment liée à la collaboration accrue entre enseignants utilisant le français, tant au niveau universitaire qu'au niveau national, grâce en particulier aux activités réalisées dans le cadre de l'IUFS. Le degré de connaissance et d'utilisation des principes de l'EMILE s'avère plus important chez les enseignants au profil linguistique, mais le critère de la langue n'a cependant pas été aussi décisif que prévu, même si nous nous attendions à ce que les enseignants utilisant l'anglais soient plus au fait des principes de l'EMILE, la majorité des programmes d'études étant proposés en anglais, ce qui se reflète également au niveau de la recherche scientifique.

Nous avons pu effectuer les mêmes constatations en analysant le degré d'intégration des langues et du contenu de spécialité. Près de la moitié des répondants affirme en effet s'attacher équitablement à l'acquisition du contenu de spécialité et à l'amélioration des compétences en langue étrangère. Cela concerne notamment la majeure partie des enseignants de spécialité utilisant le français alors que leurs collègues utilisant l'anglais se concentrent principalement sur l'acquisition du nouveau contenu de spécialité.

Les enseignants de langue étrangère, quelle que soit la langue, ont renseigné des informations plutôt homogènes, qu'il s'agisse de collaboration lors de la conception et de la réalisation d'une matière et de l'égale attention apportée au contenu de spécialité et aux compétences en langue ou concernant la délimitation de standards dans le syllabus d'une matière. Cela est dû en grande partie au fait qu'ils n'enseignent pas uniquement une langue étrangère dans leur département, mais aussi une matière de spécialité - voire une langue étrangère dans un département d'orientation non philologique. Leur formation initiale a sans aucun doute joué un rôle dans cette situation.

Dans le cadre de l'auto-évaluation, seules trois personnes ont considéré l'EMILE total et complet comme unique approche correspondant à leur pratique pédagogique. D'autres répondants ont associé cette variante à une autre possibilité : 8 l'ont combinée avec l'EMILE adjoint et 4 avec l'EMILE partiel basé sur le contenu. Environ un tiers des répondants a uniquement sélectionné l'EMILE partiel basé sur le contenu. Nous estimons que les réponses données correspondent parfaitement au profil linguistique et aux spécialités des répondants, tout en reflétant également leurs pratiques en matière d'enseignement et de recherche.

En guise de conclusion, nous souhaitons ajouter que les résultats de cette enquête par questionnaire nous ont permis de confirmer la majorité de nos hypothèses.

XLinguae, Volume 13 Issue 1, January 2020, ISSN 1337-8384, eISSN 2453-711X 
L'évaluation et l'auto-évaluation de la pratique pédagogique des enseignants interrogés démontrent l'existence d'une large palette de variantes dans le cadre de l'EMILE. Le fait que la majorité des répondants n'ait pas encore entendu parler, ne serait-ce que sur une base théorique, des approches de l'EMILE ne signifie pas nécessairement qu'ils n'en utilisent pas déjà certains principes (sous forme " douce » ou «dure ») avec des résultats certains. La plupart des enseignants familiarisés (connaissance partielle ou expérience préalable) l'ont fait de façon individuelle, plus rarement dans le cadre d'une formation initiale ou continue. Il serait donc souhaitable de se demander si la formation continue des enseignants slovaques dans ce domaine ne pourrait pas améliorer la préparation de ses étudiants, suivant ainsi l'exemple de nombreux autres pays européens. Une étude détaillée et longitudinale serait en revanche nécessaire afin de vérifier cette hypothèse.

La présente contribution a été soutenue financièrement par le projet VEGA $n^{\circ}$ 1/0437/19 : «Importance de l'internationalisation de l'enseignement supérieur pour la construction des identités de l'Union européenne et l'augmentation de la compétitivité dans l'espace européen ». 
Tableau n ${ }^{\circ} 1$ : Classification d'EMILE selon A. Greere et A. Rasanen (2008)

\begin{tabular}{|c|c|c|c|c|c|}
\hline TYPE & $\begin{array}{c}\text { PRÉ-EMILE } \\
\text { (Langue sur objectif } \\
\text { spécifique) }\end{array}$ & $\begin{array}{l}\text { EMILE PARTIEL } \\
\text { basé sur la langue }\end{array}$ & $\begin{array}{l}\text { EMILE PARTIEL } \\
\text { basé sur le contenu }\end{array}$ & EMILE ADJOINT & $\begin{array}{l}\text { EMILE } \\
\text { basé sur la dualité }\end{array}$ \\
\hline \multicolumn{6}{|c|}{ CARACTÉRISITIQUES } \\
\hline Principaux objectifs & $\begin{array}{l}\text { Maîtrise de la langue } \\
\text { et des techniques } \\
\text { d'étude requises } \\
\text { (approche } \\
\text { actionnelle) ; } \\
\text { objectifs semblables } \\
\text { à ceux d'une L2. }\end{array}$ & $\begin{array}{l}\text { Maîtrise de la langue et } \\
\text { de techniques d'étude } \\
\text { calibrées pour } \\
\text { l'apprentissage futur de } \\
\text { contenus } \\
\text { pédagogiques, ex : } \\
\text { cours à dimension } \\
\text { professionnelle ; } \\
\text { objectifs semblables à } \\
\text { ceux d'une L2. }\end{array}$ & $\begin{array}{l}\text { Maîtrise des contenus ; } \\
\text { apprentissage indirect } \\
\text { de la L2 - objectifs } \\
\text { langagiers non } \\
\text { spécifiés, mais parfois } \\
\text { implicites (semblables } \\
\text { à ceux d'une L2). }\end{array}$ & $\begin{array}{l}\text { Maîtrise des contenus } \\
\text { et apprentissage de la } \\
\text { L2 ; L2 calibré et } \\
\text { adjointe visant à } \\
\text { soutenir } \\
\text { l'apprentissage des } \\
\text { acquis ; objectifs } \\
\text { explicites de L2. }\end{array}$ & $\begin{array}{l}\text { Maîtrise des contenus } \\
\text { et apprentissage de la } \\
\text { L2 ; double } \\
\text { focalisation et objectifs } \\
\text { harmonisés et définis } \\
\text { pour la langue et la } \\
\text { spécialité. }\end{array}$ \\
\hline
\end{tabular}




\begin{tabular}{|c|c|c|c|c|c|}
\hline $\begin{array}{l}\text { Approches } \\
\text { pédagogiques }\end{array}$ & $\begin{array}{l}\text { Enseignement de } \\
\text { langue et approche } \\
\text { de LOS avec } \\
\text { attention accrue sur } \\
\text { l'approche } \\
\text { actionnelle. Tâches } \\
\text { d'apprentissage } \\
\text { calibrées. } \\
\text { Enseignement des } \\
\text { techniques d'étude et } \\
\text { focalisation } \\
\text { additionnelle sur la } \\
\text { LOS. }\end{array}$ & $\begin{array}{l}\text { Enseignement des } \\
\text { techniques d'étude et } \\
\text { approche actionnelle } \\
\text { avec focalisation } \\
\text { additionnelle sur la } \\
\text { LOS. Tâches } \\
\text { d'apprentissage } \\
\text { calibrées. }\end{array}$ & $\begin{array}{l}\text { Enseignement souvent } \\
\text { basé sur des cours } \\
\text { magistraux, centré sur } \\
\text { la transmission d'un } \\
\text { savoir du point de vue } \\
\text { d'un expert. } \\
\text { L'approche dépend de } \\
\text { la discipline et des } \\
\text { préférences } \\
\text { l'enseignant. }\end{array}$ & $\begin{array}{l}\text { Cours magistraux ou } \\
\text { centrés sur l'apprenant } \\
\text {; cours de L2 adjoints } \\
\text { et construits en } \\
\text { collaboration par des } \\
\text { spécialistes de langue } \\
\text { et du domaine de } \\
\text { spécialité afin de } \\
\text { favoriser les } \\
\text { compétences requises } \\
\text { ou la maîtrise d'un } \\
\text { contenu. }\end{array}$ & $\begin{array}{l}\text { Approches } \\
\text { multimodales, } \\
\text { interactives et centrées } \\
\text { sur l'apprenant } \\
\text { favorisant } \\
\text { systématiquement les } \\
\text { objectifs de contenu et } \\
\text { de L2. }\end{array}$ \\
\hline $\begin{array}{l}\text { Acquis/résultats } \\
\text { attendus }\end{array}$ & $\begin{array}{l}\text { Compétence de LOS } \\
: \text { langue } \\
\text { professionnelle et } \\
\text { fonctionnelle, } \\
\text { compétences } \\
\text { communicationnelles } \\
\text { générales et dans le } \\
\text { champ de la } \\
\text { spécialité. } \\
\text { Compétence de LOS } \\
\text { liée aux objectifs de } \\
\text { la discipline. }\end{array}$ & $\begin{array}{l}\text { Compétence de LOS } \\
\text { pour les besoins de la } \\
\text { discipline. Compétence } \\
\text { de LOS : langue } \\
\text { professionnelle et } \\
\text { fonctionnelle, } \\
\text { compétences } \\
\text { communicationnelles } \\
\text { générales et dans le } \\
\text { champ de la spécialité. }\end{array}$ & $\begin{array}{l}\text { Comme pour } \\
\text { l'enseignement du } \\
\text { contenu. Apprentissage } \\
\text { de la langue dépendant } \\
\text { de l'approche } \\
\text { pédagogique ainsi que } \\
\text { de la motivation, de } \\
\text { l'esprit d'initiative et } \\
\text { de l'autonomie de } \\
\text { l'apprenant. Une rare } \\
\text { prise en compte du rôle } \\
\text { de la langue est } \\
\text { fréquente. }\end{array}$ & 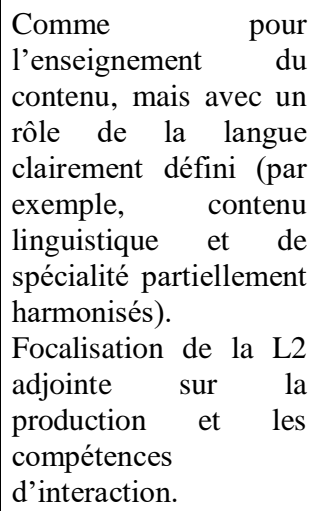 & $\begin{array}{l}\text { Contenus linguistiques } \\
\text { et de spécialité } \\
\text { harmonisés, les deux } \\
\text { étant systématiquement } \\
\text { élaborés par le biais de } \\
\text { tâches calibrées ; fort } \\
\text { accent sur } \\
\text { l'apprentissage de la } \\
\text { L2, la production et les } \\
\text { compétences } \\
\text { d'interaction. } \\
\text { Reconnaissance totale } \\
\text { de la langue. }\end{array}$ \\
\hline
\end{tabular}




\begin{tabular}{|c|c|c|c|c|c|}
\hline Évaluation & $\begin{array}{l}\text { Évaluation du niveau } \\
\text { de langue et des } \\
\text { compétences } \\
\text { communicationnelles } \\
\text { selon des critères } \\
\text { définis. }\end{array}$ & $\begin{array}{l}\text { Évaluation du niveau } \\
\text { de langue et des } \\
\text { compétences } \\
\text { communicationnelles. }\end{array}$ & $\begin{array}{l}\text { Maitrise des contenus } \\
\text { évaluée par tous les } \\
\text { moyens habituels ; } \\
\text { apprentissage de la } \\
\text { langue non évalué } \\
\text { (sauf } \text { possibilité } \\
\text { d'auto-évaluation). }\end{array}$ & $\begin{array}{l}\text { Chaque enseignant } \\
\text { évalue sa propre partie } \\
\text {; critères d'évaluation } \\
\text { souvent communs et } \\
\text { formes multiples ; } \\
\text { crédits accordés à } \\
\text { chaque partie. }\end{array}$ & 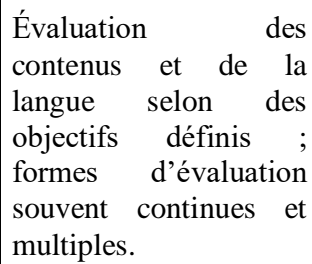 \\
\hline
\end{tabular}




\section{Bibliographic references}

ALIPICHEV, A. Y. - GALUSHKIN, A. - DRONOVA, S. Y. - PANFILOVA, S. A. 2017. Towards successful implementation of CLIL courses in Russian universities. In: XLinguae, vol. 10, n. 4, ISSN 1337-8384, eISSN 2453-711X.

ARNO-MACIA, E. - MANCHO-BARES, G. 2015. The role of content and language in content and language integrated learning (CLIL) at university: Challenges and implications for ESP. English for Specific Purposes, vol. 37, pp. 63-73.

BADINSKA, M. 2013. Subjects taught through foreign languages: innovations in the teaching of political science and international relations at Matej Bel University in Slovakia. In: Applied natural sciences. Trnava: University of SS. Cyril and Methodius in Trnava, pp. 317-321. ISBN 978-80-8105-502-7.

BADINSKA, M. 2011. CLIL (Content and Language Integrate Learning) Implemented to the Modul Slovak Studies. Trnava: University of SS. Cyril and Methodius, 2011, pp. 353-357. ISBN 978-80-8105-266-8.

BENCEKOVA, M. 2019. Rozvoj interkulturnych kompetencii ako klucova oblast edukacnej pripravy buducich diplomatov na diplomaticku prax $\mathrm{v}$ podmienkach globalizacie. In: VLCEK, D. - PAJTINKA, E. - BENCEKOVA, M. 2019. Premeny diplomacie $\mathrm{v}$ globalizovanych medzinarodnych vztahoch $\mathrm{v}$ kontexte makkej moci. Vytlacene v Tiskarna V\&H Print Hlavko s.r.o., Nove Mesto nad Metuji. ISBN 97880-206-1846-7.

CHOVANCOVA, K. 2008. Interkulturalita ako "pridana hodnota" vo vyucovacom procese. In: Europa v skole. Nitra: Enigma, pp. 181-199. ISBN 978-80-89132-58-4.

COME, T. - GUEORGUIEVA, P. - GURA, R. ROUET, G. 2018. Vers une gouvernance nouvelle de l'universite en Europe?

In: Management and governance of the University of the Future : research on competitiveness, globalisation and development. Łodz: Wydawnictwo Społecznej Akademii Nauk, pp. 109-123. ISBN 978-83-64971-47-1

COYLE, D. 2008. CLIL - A pedagogical approach from the European perspective. In: N. H. Hornberger (Ed.), Encyclopedia of Language and Education. Boston, MA: Springer US, pp. 97-111. Available online: https://doi.org/10.1007/978-0-387-304243_92

COYLE, D. - HOOD, P. - MARSH, D. 2010. CLIL: content and language integrated learning. Cambridge, Cambridge University Press.

DALTON-PUFFER, C. 2019. CLIL in practice: what does the research tell us? Goethe-Institut. Available online: https://www.goethe.de/en/spr/unt/kum/clg/20984546.html

GABILLON, Z. 2019. Enseignement d'une maticre par l'integration d'une langue etrangcre: recherche et pedagogie. In: Contexte didactique. Disponible sur: https://www.contextesetdidactiques.com/1110

GONZALEZ ARDEO, J. B. 2013. (In)compatibility of CLIL and ESP courses at university Language Value December 2013, vol. 5, n. 1, pp. 24-47. ISSN 1989-7103.

GREERE, A. - RASANEN, A. 2008. Redefining 'CLIL' - towards multilingual competence. [LANQUA subproject on Content and Language Integrated Learning]. Available online: http://www.unifg.it/

GURA, R. - ROSTEKOVA, M. 2018. Francuzsky model profesionalizacie terciarneho vzdelavania na Slovensku. In: Politicke vedy. vol. 21, n. 3, 2018, pp. 236244. ISSN $1335-2741$.

GURA, R. 2005. Praca s odbornym pisanym textom. In: Sbornik prispevku z konference Profilingua 2005. Plzen: Zapadoceska univerzita, pp. 114-118. ISBN 807043-424-4.

HANESOVA, D. 2014. Development of critical and creative thinking skills in CLIL. In: JoLaCE: journal of language and cultural education. Nitra: SlovakEdu, 2014. vol. 2, n. 2, pp. 33-51. ISSN 1339-4045. 
HANESOVA, D. History of CLIL. In CLIL in foreign language education: e-textbook for foreign language teachers. Nitra: Constantine the Philosopher University, 2015, pp. 7-16. ISBN 978-80-558-0889-5.

HOREHAJOVA, M. - MARASOVA, J. 2017. History and transformations of francophone study. In: Economic theory and practice 2017, proceedings of the Faculty of Economics of Matej Bel University in Banska Bystrica, Volume I. Banska Bystrica: Vydavatelstvo Univerzity Mateja Bela, Belianum, 2018. ISBN 978-80-5571424-0.

HURAJOVA, A. 2017. Contribution of CLIL Methodology to the Development of Bilingualism and Bilingual Language Competence of Slovak Secondary-School Students. European Journal of Educational Research vol. 8, n. 4, pp. 905 - 919. ISSN: 2165-8714.

KRESAKOVA, V. - PECNIKOVA, J. 2019. Inovativne metody vo vyucbe kulturnych studii a cudzich jazykov. In: Forlang 2019 : cudzie jazyky v akademickom prostredi. Kosice: Technicka univerzita v Kosiciach, pp. 257-266. ISBN 978-80-5533398-4. ISSN 1338-5496.

KUBEKOVA, J. 2017. Komparacia viacslovnych ustalenych spojeni v odbornom jazyku s priznakom farby medzi spanielcinou a slovencinou. In: Od textu k prekladu XII. Praha: Jednota tlumocniku a prekladatelu, pp. 19-29. ISBN 978-80-7374-126-6.

LIPS, C. 2016. L'appropriation du Processus de Bologne par les acteurs de l'universite, enjeux et perspectives. These de doctorat en Sciences de gestion. Institut Superieur de Management, Universite Versailles-Saint-Quentin, Paris Saclay.

LUPRICHOVA, J. - HURAJOVA, L. 2017. CLIL-Potential Way of Enhancing Internationalization of Higher Education in Diverse Context. Available online: https://www.researchgate.net/publication/320756077_CLIL-

Potential_Way_of_Enhancing_Internationalization_of_Higher_Education_in_Diverse Context

MARTIN DE LAMA, M. T. 2015. Making the Match between Content and Foreign Language: A Case Study on University Students' Opinions towards CLIL. In: Higher Learning Research Communications. vol. 5, n. 1, pp. 29-46.

MOLNAROVA, E. 2016. Odborny jazyk vo vzdelavani buducich ucitelov nemeckeho jazyka. In Implementacia edukacnych metod pre skvalitnenie vyucovacieho procesu $\mathrm{v}$ oblasti Technickej mechaniky. Zvolen: Technicka univerzita, 2016, pp. 75-80. ISBN 978-80-228-2875-8.

MORTREUIL, F. X. 2017. Prihovor na slavnostnom odovzdavani diplomov na EF UMB 15. novembra 2017.

NAPOLI, J. - SOURISSEAU, J. 2013. L'EMILE est-il applique dans l'enseignement superieur? In: Recherche et pratiques pedagogiques en langues de specialite [Online], Vol. XXXII N 3/2013.

PALOVA, M. - ZELENAKOVA, M. 2018. We Are Looking for Graduates with Professional Experience: Forum Students - Enterprises 2018 Offers a Solution to the Slovak Paradox. In: Politicke vedy. vol. 21, n. 2, 2018, pp. 203-210. ISSN 1335 2741.

PEREZ-CANADO, M. L. 2013. International Journal of Bilingual Education and Bilingualism CLIL research in Europe: past, present and future. In: International Journal of Bilingual Education and Bilingualism, vol 15, n. 3, pp. 315-341.

POKRIVCAKOVA, S. 2015. CLIL in Slovakia: projects, research, and teacher training (2005-2015). In: CLIL in Foreign Language Education. Nitra: UKF, pp. 1729.

POLCICOVA, M. 2018. Didakticke vyzvy obraznosti vo vyucbe odbornej komunikacie. In: QUAERE 2018. Hradec Kralove: Magnanimitas, pp. 782-790. ISBN 978-80-87952-26-9.

XLinguae, Volume 13 Issue 1, January 2020, ISSN 1337-8384, eISSN 2453-711X 
RASANEN, A. - KLAASSEN, R. 2006. From learning outcomes to staff competences in integrated content and language instruction at the higher education level. In: WILKINSON, R. - ZEGERS, V. - van LEEUWEN, C. (eds.). Bridging the Assessment Gap in English-medium Higher Education. AKS-Series: Fremdsprachen in Lehre und Forschung. Bochum: AKS-Verlag, pp. 256-280.

ROSTEKOVA, M. 2018. Francuzsko-slovenska spolupraca ako motor internacionalizacie vysokoskolskeho vzdelavania na Slovensku. In: Politicke vedy. vol. 21, n. 1, 2018, pp. 204-211. ISSN 1335 - 2741.

SCHMITT, F. 2019. Le modcle philologique dans la perspective actionnelle du français langue etrangcre. In: Synergies Europe, n. 14, Sylvains les Moulins : Gertflint, pp. 63-76. ISSN 1951-6088.

SOLTES, V. - NEUPAUEROVA, M. 2008. Francuzsky studijny program na Technickej univerzite $\mathrm{v}$ Kosiciach, Ekonomickej fakulte $\mathrm{v}$ kontexte integracie europskeho vzdelavania. In: Lisbon Strategy as a Determinant Factor of European Integration in the Sphere of Education and Science. Uzhorod: ZDU, pp. 28-35. ISBN 9789662195323.

ZELENKOVA, A. - SPISIAKOVA, M. 2009. The development of ESP: a case of the Faculty of economics, MBU Banska Bystrica. In: Acta Linguistica No 7: languages for specific purposes and intercultural communication. Banska Bystrica: Univerzita Mateja Bela, Ekonomicka fakulta, pp. 315-320. ISBN 978-80-8083-791-4.

Words: 9494

Characters: 66450 (36,92 standard pages)

PaedDr. Maria Rostekova, PhD.

Department of International Relations and Diplomacy

Faculty of Political Science and International Relations Matej Bel University in

Banska Bystrica

Kuzmanyho 197401

Banska Bystrica

Slovakia

PhDr. Maria Palova, PhD.

Department of British and American Studies

Faculty of Arts Pavol Jozef Safarik University in Kosice

Moyzesova 904001

Kosice

Slovakia 